\title{
Lower-limb amputees can reduce the energy cost of walking when assisted by an Active Pelvis Orthosis*
}

\author{
E. Martini, C.B. Sanz-Morère, C. Livolsi, A. Pergolini, G. Arnetoli, S. Doronzio, A. Giffone, R. Conti, \\ F. Giovacchini, P. Friðriksson, K. Lechler, S. Crea and N. Vitiello, Member, IEEE
}

\begin{abstract}
Exoskeletons could compete with active prostheses as effective aids to reduce the increased metabolic demands faced by lower-limb amputees during locomotion. However, little evidence of their efficacy with amputees has been provided so far. In this paper, a portable hip exoskeleton has been tested with seven healthy subjects and two transfemoral amputees, with the final goal to verify whether a hip flexion-extension assistance could be effective in reducing the metabolic cost of walking. The metabolic power of the participants was estimated through indirect calorimetry during alternated repetitions of three treadmill-based walking conditions: without the exoskeleton (NoExo), with the exoskeleton in zero-torque mode (ExoTM) and with the exoskeleton providing hip flexion-extension assistance (ExoAM). The results showed that the exoskeleton reduced the net metabolic power of the two amputees in ExoAM with respect to NoExo, by $5.0 \%$ and $3.4 \%$. With healthy subjects, a $5.5 \pm 3.1 \%$ average reduction in the metabolic power was observed during ExoAM compared to ExoTM (differences were not statistically significant), whereas ExoAM required $3.9 \pm 3.0 \%$ higher metabolic power than NoExo (differences were not statistically significant). These results provide initial evidence of the potential of exoskeletal technologies for assisting lower-limb amputees, thereby paving the way for further experimentations.
\end{abstract}

\section{INTRODUCTION}

Data on the incidence of lower-limb loss report that most amputation cases are performed on elderly patients with

${ }^{*}$ Research supported by the European Commission under the CYBERLEGs Plus Plus project (grant $\mathrm{n}^{\circ}$ 731931), within the H2020 framework (H2020-ICT-25-2016-2017).

E. M., C.B. S.M., C. L., A. P., S. C. and N. V. are with The BioRobotics Institute, Scuola Superiore Sant'Anna, 56127 Pisa, Italy (e-mail: elena.martini@santannapisa.it, clarabeatriz.sanzmorere@santannapisa.it, chiara.livolsi@santannapisa.it, $\quad$ andrea.pergolini@ santannapisa.it, nicola.vitiello@santannapisa.it, simona.crea@santannapisa.it). S. C. and N. V. are also with IRCCS Fondazione Don Carlo Gnocchi, 20148 Milan, Italy and the Department of Excellence in Robotics \& AI, Scuola Superiore Sant'Anna, 56127 Pisa, Italy. F. G., R. C. and C. L. are with IUVO S.r.l, Via Puglie, 9, 56025 Pontedera (PI), Italy (e-mail: francesco.giovacchini@iuvo.company, roberto.conti@iuvo.company, chiara.livolsi@iuvo.company). P. F. and K. L. are with Össur, Grjótháls 5, 110 Reykjavík (Iceland) (e-mail: thfridriksson@ ossur.com, klechler@ossur.com). G. A., S. D. and A.G. are with IRCCS Fondazione Don Carlo Gnocchi, 20148 Milan, Italy (e-mail: gabri.arnetoli@gmail.com, doronziostefano@gmail.com, antonella.giffone@ hotmail.it).

E.M. is the corresponding author (phone: +39 050883164; e-mail: elena.martini@santannapisa.it).

F.G., R.C., S.C. and N.V. have interests in IUVO Srl (Pontedera, Italy). Part of the IP covering the technology presented in this paper has been exclusively licensed to IUVO for commercial exploitation. limited general health and fitness [1]. Current passive or semi-active prostheses do not completely restore physiological gait patterns and, as a consequence, amputees typically spend $30-60 \%$ more energy compared to ablebodied individuals [2], [3], with negative effects on health, independence and quality of life [1], [4], [5].

Active prostheses represent a widely pursued solution for recovering amputees' gait and functional abilities beyond what currently allowed by passive prostheses. However, the considerable distal weight of a powered knee-ankle prosthesis may not be optimal to reduce the metabolic cost of walking in transfemoral amputees [4]-[7]. Exoskeleton solutions on the other hand, have rarely been explored in applications related to amputees gait assistance [8], [9]. Thus far, the assistance of a quasi-passive knee orthosis coupled to a powered ankle prosthesis has been shown to account for $3-5 \%$ metabolic reduction in two transtibial amputees [10]. In [6], the authors have hypothesized that, since hip and ankle equally contribute to the average positive power produced by the lower limb throughout the stride [11], hip exoskeletons could outperform active prostheses in improving locomotion efficiency of transfemoral amputees, given their proximal weight distribution; in that study a unilateral hip device was shown to lower the Physiological Cost Index (PCI) in one transfemoral amputee.

Except for the promising results of the aforementioned early works, the authors of this study are not aware of other studies exploring the metabolic effects of exoskeleton assistance for amputees.

In this study, we investigated the effects of a portable hip exoskeleton on metabolic consumption on a convenience sample of participants, including two transfemoral amputees and seven healthy subjects, in three walking tasks. Namely, the tasks were: (i) natural walking (NoExo), (ii) walking with the exoskeleton controlled in transparent mode, i.e. providing zero output torque (ExoTM) and (iii) walking with bilateral flexion-extension assistance (ExoAM).

\section{MATERIALS AND METHODS}

\section{A. The Active Pelvis Orthosis}

The Active Pelvis Orthosis (APO) is a powered exoskeleton that assists hip flexion/extension during locomotion. The APO used in this study is an evolution of the prototype described in [12], which is now fully portable and weights approximately $5 \mathrm{~kg}$. The control electronics and the 
battery operating the system are housed in a backpack secured to the user's body by means of an orthotic shell, designed to unload most of the device weight on the iliac crests, to minimally affect walking energetics [13]. Two actuation units located in the lower part of the backpack transfer the power via a 4-bar mechanism to the corresponding hip joints, which are located in line with the flexion-extension rotation axes of the biological hip joints. Two output links connect the device to the posterior part of the user's thighs by means of adjustable straps. The hip motion in the sagittal plane has a range of $[-20,100]$ deg (positive values in flexion). In order to avoid hindering the movement in the frontal plane, a passive abduction/adduction mechanism has been integrated into the device structure, with a range of $[-15,30]$ deg (positive values in abduction). Each actuation unit implements a Series Elastic Actuator (SEA) architecture, including a 70W DC motor (EC45 flat, Maxon Motor®, Sachseln, Switzerland), a gear reduction stage (Harmonic Drive, 100:1) and a custom torsional spring ( 200 $\mathrm{Nm} / \mathrm{rad}$ ). The actuation units have been designed to provide torque peaks of $\sim 15 \mathrm{Nm}$ and minimal mechanical output impedance when the device is controlled in transparent mode, i.e. it provides null output torque (previous characterizations resulted in a mechanical output impedance of $\sim 0.5 \mathrm{Nm} / \mathrm{rad}$ at $1 \mathrm{~Hz}$ ). The control system runs on a NI SbRIO9651 processor (National Instruments ${ }^{\mathrm{TM}}$, USA) featured with both a FPGA and Arm controllers. Two absolute 17-bits encoders are used to estimate the user hip angle and to read the spring deformation, the latter necessary to measure the output torque and close the low-level torque control loop. The hip angle is used by the high-level controller, which estimates the gait phase based on adaptive oscillators and locks the $0 \%$ of the gait cycle to the flexion peak of the hip angle [14]. Assistive torque profiles are computed according to different strategies. For this study, the so-called virtual stiffness and feed-forward assistive strategies were used. The former estimates the assistive torque using an impedance control strategy based on the hip angular profile and the predicted profile calculated using nonlinear Kernel filter coupled to adaptive oscillators [15]. The second one provides phase-locked torque profiles, shaped as Gaussian curves tunable in amplitude, phase and duration. Both modalities are illustrated in more detail in Figure $1 \mathrm{~b}$.

\section{B. Participants}

A convenience sample of two transfemoral amputees (identified as IDA and IDB) and seven healthy volunteers (identified as IDH1-7) took part in the experiments (Table I). The trials were conducted according to local regulations and with the approval of the reference Ethics Committee (Ethics Committee of Area Vasta Centro Toscana, approval number: 12739_spe) and provision of participants' written informed consent.

\section{Experimental Procedures}

Subjects participated to two trials, namely a familiarization and an assessment trial. The familiarization was intended to warm-up, familiarize users with the device and tune the assistive parameters; the assessment was devoted to assessing metabolic consumption in different experimental conditions. The familiarization was performed based on the following sequence. First, treadmill speed was selected. For healthy participants, gait speeds ranged between 0.94 and $1 \mathrm{~m} / \mathrm{s}$; experiments were conducted with $+6 \%$ incline, in order to

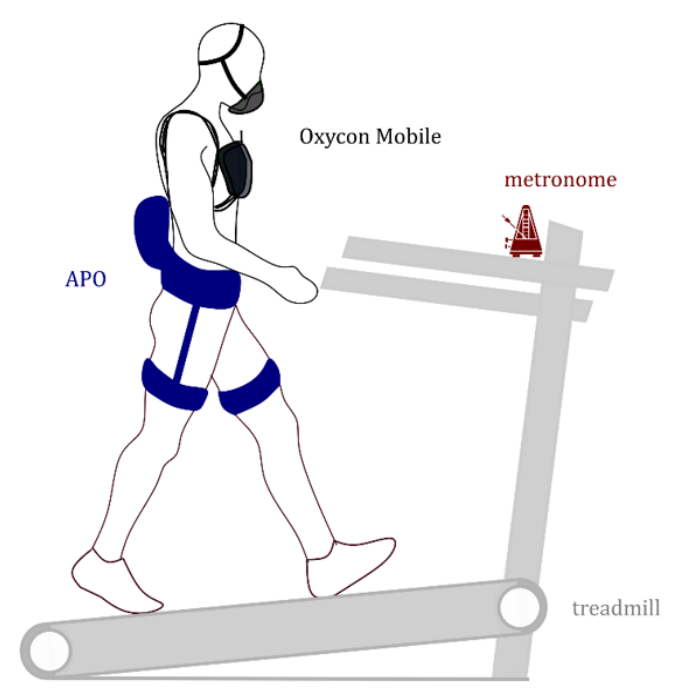

(a)

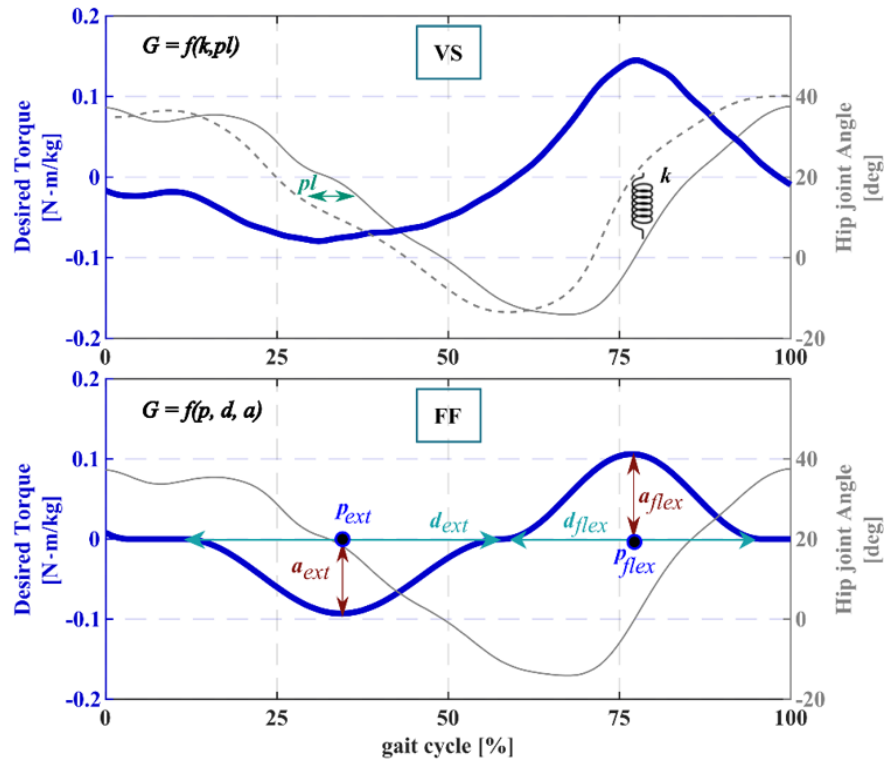

(b)

Figure 1. (a) Experimental setup: participants walked on a treadmill (i) without the APO (NoExo), (ii) with the APO in zero-torque mode (ExoTM) and (iii) with the APO providing assistive torque (ExoAM); during the assessment trial, a metronome was used to keep cadence constant and the Oxycon Mobile gas analyzer recorded the rated of $\mathrm{O}_{2}$ and $\mathrm{CO}_{2}$ consumptions. (b) Sample assistive torque profiles for the virtual stiffness (VS) and feed-forward (FF) strategies. In VS, the assistive torque is proportional to the difference between the predicted (dashed line) and the measured (solid line) hip joint angles by means of the proportional constant k, called "virtual stiffness". The predicted joint angle is the hip angle estimated by the adaptive oscillators with a phase shift equal to the set "phase lead" (pl); the measured hip angle is given by the reading of the hip joint encoder [14],[15]. In FF, extensor and flexor torques are shaped as Gaussian curves. The operator decides the phase of the torque peak ( $\mathrm{p} \%$ of the gait cycle]), the duration of the assistance (d [\% of the gait cycle]) and its amplitude (a [Nm]) separately for flexor and extensor torques. 
increase the exercise intensity and lead to energetic expenditure closer to the typical values experienced by amputees. Incline walking was preferred over walking at high speed in order to remain within the range of optimal performance of the APO. Amputees selected their comfortable treadmill speed (Table I), as done in similar studies [16], [17], and walked with a slope of $+3 \%$. Once treadmill settings had been adjusted, the participants donned the APO and started walking while the operator tuned the assistive parameters (Figure 1b): torque was provided bilaterally, during both the extension and flexion phases, using either the virtual stiffness or the feed-forward strategies and torque peaks were tuned to reach $\sim 10 \%$ of the biological hip torque, estimated considering the user's body mass [18]. In general, the virtual stiffness mode was considered first, as it allows a simpler and faster tuning procedure. However, as the assistive profiles resulting from this strategy are highly dependent on the accuracy and repeatability of the hip angular profiles -which in turn may be affected by a suboptimal device wearability- the feed-forward strategy was preferred for users whose hip angular profiles were not deemed reliable when tuning the virtual stiffness mode. For the feed-forward strategy, torque timing and duration were set according to the torque profiles that had resulted from the virtual stiffness algorithm, in order to provide comparable torque patterns across users regardless of the selected assistive strategy (Table I). After setting the parameters, the subjects continued walking while alternating between $\sim 3$ min of "assisted" (ExoAM) and "transparent" (ExoTM) conditions. For amputees, short rests were allowed between conditions, to prevent excessive fatigue, determined by $\mathrm{HR}>80 \% \mathrm{HR}_{\max }$ or respiratory exchange ratios $(\mathrm{RER})>1.1$ [19]. Heart rate and $\mathrm{O}_{2} / \mathrm{CO}_{2}$ exchange rates were monitored throughout the trial using a portable gas analyzer (Oxycon Mobile, CareFusion, Germany) and under the supervision of a medical doctor, to control exercise intensity and to verify the effectiveness of ExoAM versus ExoTM. In the last
ExoAM condition during familiarization, subjects' stride period was measured online (as the time between consecutive hip flexion peaks) and used to set a metronome to minimize cadence variations between the ExoAM and ExoTM conditions [20]. At the end of the familiarization, the participants were allowed to rest $\sim 30 \mathrm{~min}$. The assessment trial started when stress indicators (blood pressure, oxygen saturation and HR) had returned to baseline levels. The assessment trial consisted of three walking conditions, namely NoExo, ExoAM and ExoTM; all conditions were conducted on the treadmill at the previously selected speed and with a metronome to keep a fixed cadence. Each condition had a duration comprised between 3 and 6 minutes, depending on the required time to reach the steady state, indicated by a plateau observed by the experimenters in the oxygen uptake rate. Indeed the plateau is generally reached after $\sim 3$ min of submaximal activity [21], but during prolonged exercise without breaks or with just little recovery, shorter adaptation times were considered ( 1 or 2 minutes). As in the familiarization trial, heart rate and the rates of $\mathrm{O}_{2}$ uptake and $\mathrm{CO}_{2}$ output were monitored by physical therapists and a medical doctor. For amputees, baseline consumptions (BL) were recorded prior to start walking in the standing position and each condition was followed by a short resting period while standing still. Trial sequences and durations are provided in Table II.

\section{Data Analysis}

During the assessment trial, the heart rate, $\mathrm{O}_{2}$ uptake and $\mathrm{CO}_{2}$ output were recorded by the gas analyzer and exported from the system software on a temporal basis $(0.2 \mathrm{~Hz})$, while the APO acquired the hip angle and torque data at $100 \mathrm{~Hz}$. The data were stored and analyzed offline in Matlab (Mathworks Inc., Natick, USA).

Metabolic data were filtered (mobile median filter, 5th order), normalized by body mass and used to compute the

TABLE I. SUMMARY OF THE CHARACTERISTICS OF THE PARTICIPANTS, TREADMILL SPEEDS, SLOPES AND ASSISTIVE STRATEGIES

\begin{tabular}{|c|c|c|c|c|c|c|c|c|c|c|c|c|}
\hline \multirow[t]{2}{*}{ Subject } & \multirow{2}{*}{$\begin{array}{c}\text { Medicare } \\
\text { functional } \\
\text { level }\end{array}$} & \multirow{2}{*}{$\begin{array}{l}\text { Amputation } \\
\text { side }\end{array}$} & \multicolumn{2}{|c|}{ Prosthesis type } & \multirow[t]{2}{*}{ Sex } & \multirow[t]{2}{*}{ Age } & \multirow{2}{*}{$\begin{array}{c}\text { Weight } \\
\text { (kg) }\end{array}$} & \multirow{2}{*}{$\begin{array}{c}\text { Height } \\
\text { (cm) }\end{array}$} & \multirow{2}{*}{$\begin{array}{c}\text { Speed } \\
(\mathrm{m} / \mathrm{s})\end{array}$} & \multirow[t]{2}{*}{ Slope } & \multirow{2}{*}{$\begin{array}{l}\text { Metronome } \\
\text { frequency } \\
\quad(\text { bpm })\end{array}$} & \multirow{2}{*}{$\begin{array}{c}\text { Assistive } \\
\text { strategy }\end{array}$} \\
\hline & & & Knee & Ankle & & & & & & & & \\
\hline IDA & $\mathrm{K} 3$ & $\mathrm{R}$ & $\begin{array}{l}\text { C-leg 3, } \\
\text { Ottobock }\end{array}$ & $\begin{array}{c}\text { Triton, } \\
\text { Ottobock }\end{array}$ & M & 72 & 66.9 & 180 & 0.78 & $3 \%$ & $44 *$ & $\mathrm{FF}$ \\
\hline IDB & $\mathrm{K} 3$ & $\mathrm{~L}$ & $\begin{array}{c}\text { 3R45, } \\
\text { Ottobock }\end{array}$ & $\begin{array}{l}\text { Energy } \\
\text { release }\end{array}$ & M & 53 & 76.0 & 166 & 0.72 & $3 \%$ & 86 & $\mathrm{FF}$ \\
\hline IDH1 & - & - & - & - & M & 26 & 67.5 & 170 & 0.94 & $6 \%$ & 105 & VS \\
\hline IDH2 & - & - & - & - & M & 26 & 73.4 & 177 & 0.94 & $6 \%$ & $50 *$ & VS \\
\hline IDH3 & - & - & - & - & M & 24 & 70.0 & 175 & 1.00 & $6 \%$ & $50 *$ & VS \\
\hline IDH4 & - & - & - & - & M & 28 & 74.1 & 170 & 1.00 & $6 \%$ & $53 *$ & $\mathrm{FF}$ \\
\hline IDH5 & - & - & - & - & M & 26 & 79.1 & 184 & 1.00 & $6 \%$ & $52 *$ & VS \\
\hline IDH6 & - & - & - & - & M & 28 & 75.3 & 175 & 1.00 & $6 \%$ & 98 & FF \\
\hline IDH7 & - & - & - & - & M & 26 & 78.0 & 165 & 1.00 & $6 \%$ & 104 & $\mathrm{FF}$ \\
\hline
\end{tabular}

TABLE II. SEQUENCE OF THE ASSESSMENT TRIAL

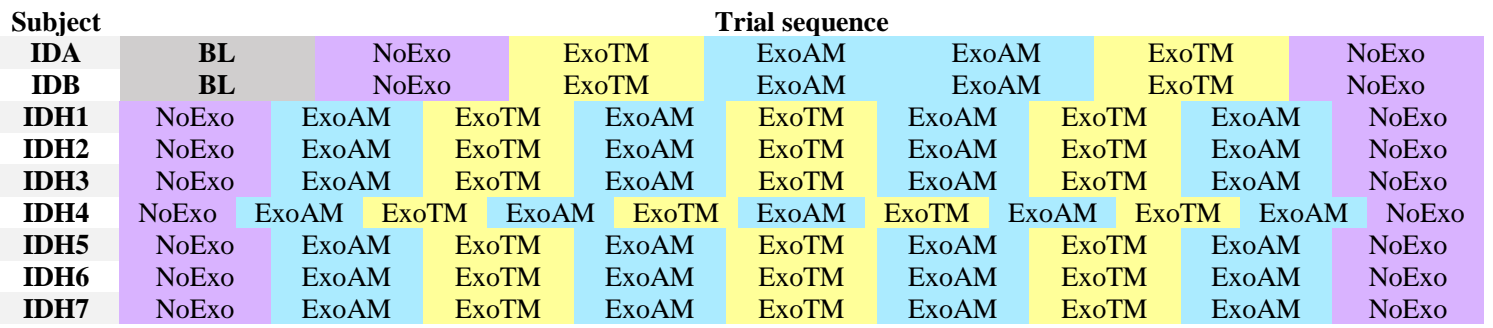


metabolic power according to the Brockway equation [22]. Nitrogen contribution was omitted from the calculation, since protein metabolism was not deemed to be relevant during the performed submaximal and relatively short exercise [17], [23]. For amputees, the net value of the metabolic power was computed by subtracting the average baseline consumptions. The values of the last two minutes of each repetition of the three conditions (ExoAM, ExoTM, NoExo) were averaged to obtain the mean steady-state values. The mean steady state values of repetitions of the same condition were further averaged to obtain the overall values for each one of the three conditions for each subject. For healthy subjects, individual values were also averaged across participants and the aggregated results were tested for significant variations among the three conditions with non-parametric ANOVA (Friedman's test, $\mathrm{p}=0.05$ ) and multiple comparison analysis (Dunn's test). The relative differences in the metabolic power between pairs of conditions were also computed; in particular, the percentage differences between (i) ExoAM and ExoTM, (ii) ExoAM and NoExo and (iii) ExoTM and NoExo were computed as:

$$
P_{\text {diff }}[\%]=\frac{P_{c 1}-P_{c 2}}{P_{c 2}} 100 ;
$$

$(c 1, c 2) \in\{($ ExoAM, ExoTM $),($ ExoAM, NoExo $),($ ExoTM , NoExo $)\}$.

Heart rate data were used to compute the Heart Rate Reserve (HRR) [24][25], as a further indicator of the individual effort sustained by amputee subjects during the trial.

As for the data recorded by the APO, the hip joint angle data were derived and filtered ( $2^{\text {nd }}$ order, Butterworth low- pass filter at $15 \mathrm{~Hz}$ ) to extract the hip velocity, which was used to estimate the power delivered by the APO. The data were segmented into single strides using the hip angle flexion peaks. For each stride, the stride period, the mean power $(\mathrm{W} / \mathrm{kg})$ and the peak flexor torque $(\mathrm{Nm} / \mathrm{kg})$ were computed and then averaged across all the strides in ExoAM and ExoTM separately. Moreover, single stride profiles were interpolated and averaged to obtain the mean patterns under ExoAM and ExoTM.

\section{RESULTS}

Figure 2a reports the oxygen uptake rates during the assessment trial for the two transfemoral amputees. When testing the APO, both subjects showed lower net metabolic power in ExoAM relatively to NoExo condition, with reductions equal to $5.0 \%$ (IDA) and $3.4 \%$ (IDB). The ExoTM condition resulted in $+2.5 \%$ and $+4.8 \%$ increases relatively to NoExo, for IDA and IDB respectively. As for exercise intensity, the NoExo condition resulted in $27.3 \pm 3.7 \%$ of HRR for IDA and $47.9 \pm 7.9 \%$ of HRR for IDB.

Similarly to amputee participants, healthy subjects walked on average with $5.5 \pm 3.1 \%$ lower metabolic power in ExoAM than in ExoTM (Figure 2b). In this case however, the ExoTM condition resulted in $10.0 \pm 4.2 \%$ higher energetic expenditure with respect to the NoExo condition (Figure $2 b$ ). Friedman's test highlighted significant differences among the three conditions $(\mathrm{p}=9.1 \mathrm{e}-4)$. The difference between ExoTM and NoExo was statistically significant $(\mathrm{p}=5.5 \mathrm{e}-4)$
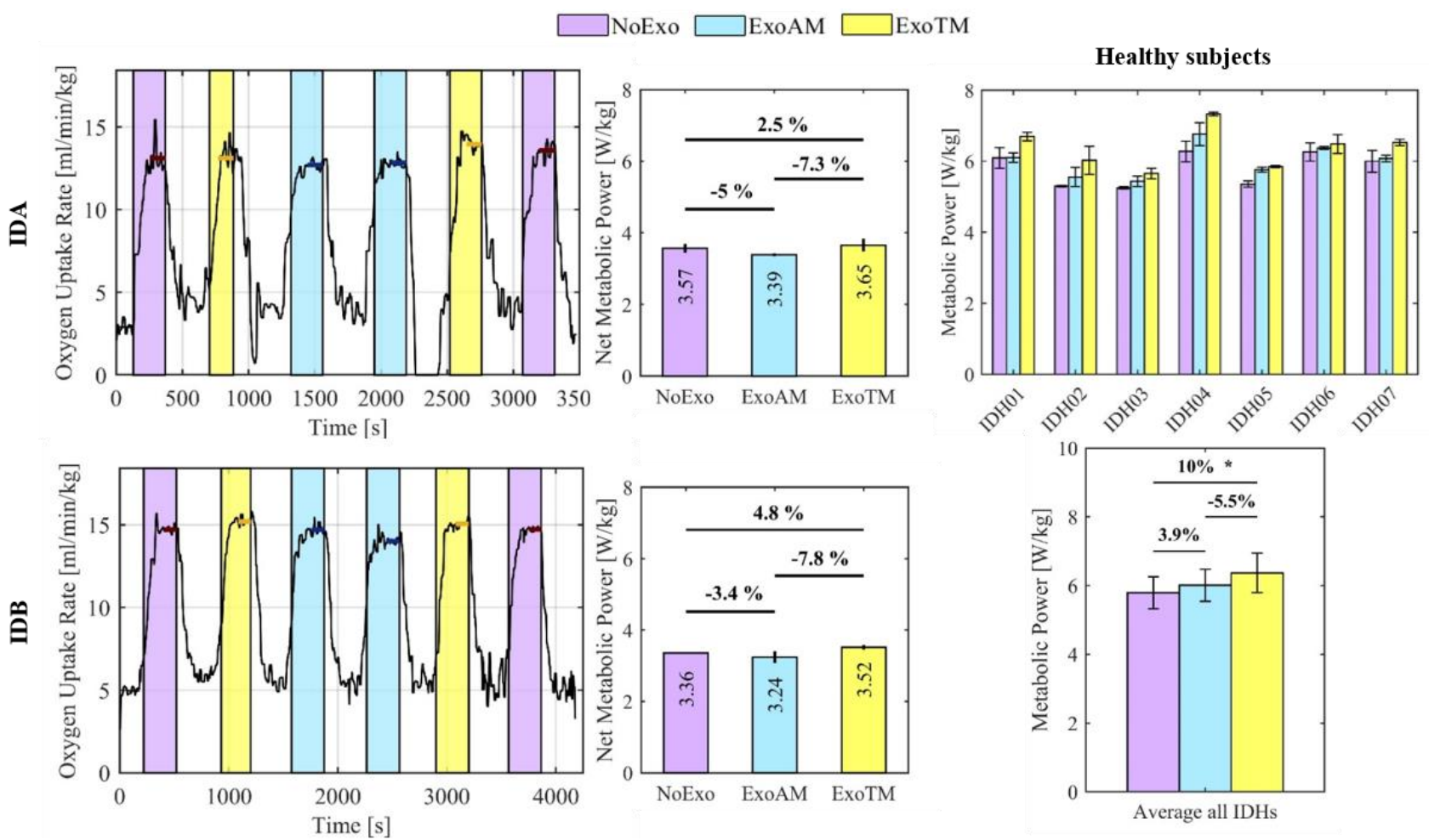

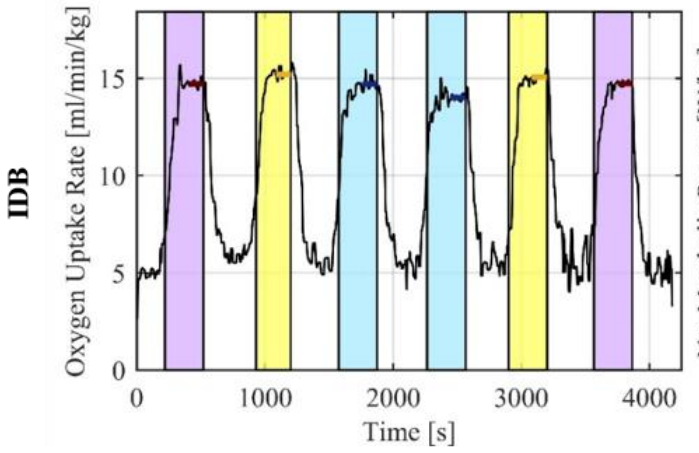

(a)

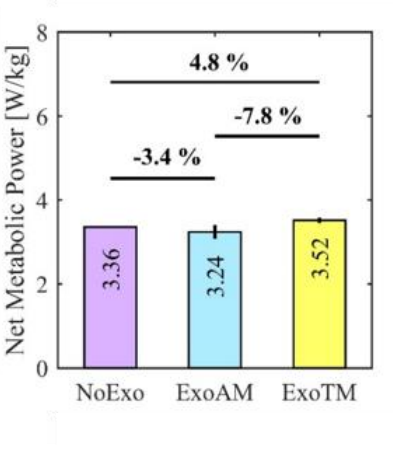

Figure 2. (a) Amputees' results: raw oxygen uptake rates recorded during the assessment trial of amputee subjects (left) and net metabolic power at the steady state for the three walking conditions; the resulting values were the average of the two repetitions of each condition. (b) Healthy subjects' results: individual metabolic power at the steady state for the three walking conditions (the resulting values were the average of the repetitions of each condition) (top); and aggregated results averaged across subjects (the star marks the only significantly different condition ( $<<0.05)$ ) (bottom). 
while the improvement between ExoTM and ExoAM was not significant $(\mathrm{p}=0.17)$.

In ExoAM, the power transferred by the APO to each hip was estimated to be on average $0.091 \pm 0.025 \mathrm{~W} / \mathrm{kg}$ for healthy subjects, $0.075 \pm 0.011 \mathrm{~W} / \mathrm{kg}$ for IDA and $0.086 \pm 0.015 \mathrm{~W} / \mathrm{kg}$ for IDB, with average peak flexor torques of $0.114 \pm 0.011 \mathrm{Nm} / \mathrm{kg}$ for healthy subjects, $0.135 \pm 0.005 \mathrm{Nm} / \mathrm{kg}$ for IDA and $0.123 \pm 0.006 \mathrm{Nm} / \mathrm{kg}$ for IDB (Table III). Average angle, torque and power profiles for IDA and IDB are shown in Figure 3.

\section{DISCUSSION}

The hip flexion/extension torque provided by the APO effectively reduced the metabolic cost of treadmill walking of the two amputees. Indeed, the limited difference of the metabolic consumption between the NoExo and ExoTM conditions allowed to observe an overall positive effect of the assistance, also when compared to walking without the exoskeleton. For gait efficiency, results of this study are comparable to those obtained in a previous study in which walking with an active knee prosthesis (weight $3.6 \mathrm{~kg}$ ) was found to decrease the metabolic power of an amputee by $6.8 \%$ with respect to a passive prosthesis [17]. Another study reported that one transfemoral amputee walking with a unilateral hip exoskeleton was able to decrease the PCI compared to walking without the exoskeleton [6]. In [6], the assistive strategy consisted of a flexor torque on the amputated side similar to the one used in this study: torque profiles were tuned with a peak equal to $0.09 \mathrm{Nm} / \mathrm{kg}$ at $58 \%$ of the gait phase (with $0 \%$ of the gait phase at the heel-
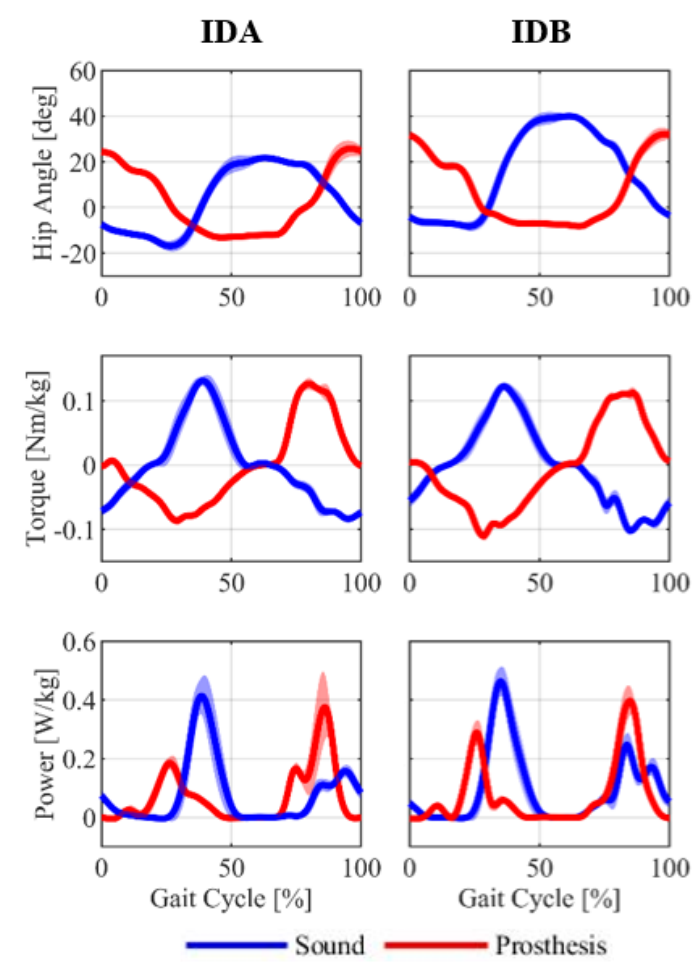

Figure 3. Hip angular profiles, APO output torque and power in ExoAM measured by onboard sensors for the two amputee subjects. strike). In line with previous research, the preliminary results obtained in this study suggest that providing flexor torque starting during the push-off with an amplitude of $\sim 0.1$ $\mathrm{Nm} / \mathrm{kg}$ could be effective to improve the gait efficiency of transfemoral amputees. Unfortunately however, the multiple differences of the two studies such as the weight and functionality of the device, the different assessment metrics and the limited number of test subjects, limit meaningful quantitative comparisons of energetic results.

Heart rate monitoring provided an additional measure of the individual fatigue faced during walking by each amputee -hence an insight on their individual fitness levelsrevealing that the same task resulted in different exercise demands for the two participants: the intensity of the NoExo condition was "low" for IDA and "moderate" for IDB, according to HRR\% reference ranges [26]. Thus the APO seemed to reduce the energetic demand of walking over a range of aerobic levels. Ideally, this ability might allow the APO to lower barriers to physical activity of deconditioned individuals by reducing the effort needed initially and adjusting based on individual fitness [27], [28]. To this end, and given the previous considerations on the effects of added load on energetic consumptions, further investigations will focus on quantifying the maximum ExoAM-vs-NoExo gain that can be expected when the added mass is kept to a minimum -e.g. detaching the actuation units from the backpack- as a "best-case" scenario.

As regards healthy subjects, the results of this study showed lower energetic expenditures in ExoAM than in ExoTM, yet the beneficial effect of the assistive action was not sufficient to cancel the added payload of carrying the exoskeleton, as the metabolic cost in ExoAM resulted higher than in the NoExo condition. In part, limited metabolic cost reductions could be explained by the short time for adaptation to the exoskeleton assistance, as longer practice seems to be necessary for optimizing the metabolic cost of walking with assistive robotic devices [29], [30]. Moreover, as a main difference with respect to amputees, healthy subjects walking with the APO in ExoTM resulted in a significantly higher increase of the metabolic effort compared to the NoExo condition. An explanation for such a different loading effect of the APO may be related to the additional anti-gravitational work necessary during slope walking, which was set to $6 \%$ for healthy subjects against the $3 \%$ selected for amputees [31]. Inclined walking was

TABLE III.

\begin{tabular}{|lccc}
\multicolumn{1}{|c|}{ ExoAM } & $\begin{array}{c}\text { Stride } \\
\text { Period }[\mathbf{s}]\end{array}$ & $\begin{array}{c}\text { APO Peak } \\
\text { Flex Torque } \\
{[\mathbf{N m} / \mathbf{k g}]}\end{array}$ & $\begin{array}{c}\text { APO Power } \\
{[\mathbf{W} / \mathbf{k g}]}\end{array}$ \\
\hline $\begin{array}{l}\text { IDA } \\
\text { (mean } \pm \mathrm{sd} \text { ) }\end{array}$ & $1.36 \pm 0.08$ & $0.135 \pm 0.005$ & $0.075 \pm 0.011$ \\
$\begin{array}{l}\text { IDB } \\
\text { (mean } \pm \mathrm{sd})\end{array}$ & $1.40 \pm 0.10$ & $0.123 \pm 0.006$ & $0.086 \pm 0.015$ \\
$\begin{array}{l}\text { IDHs } \\
\text { (mean } \pm \text { sd } \\
\text { across subj) }\end{array}$ & $1.19 \pm 0.04$ & $0.114 \pm 0.011$ & $0.091 \pm 0.025$ \\
\hline
\end{tabular}


performed in order to increase the effectiveness of the APO assistance, as uphill walking has been shown to require a greater relative contribution of the hip joint to the overall positive power of the lower-limb than level walking [32]. However, the benefit brought by the assistance remained lower than generally achieved with other hip devices during level walking and delivering lower or comparable power with similar torque timings [33]-[35]. Different assessment conditions across studies such as different loading conditions, treadmill speed and inclinations, as well as methodical variations in the computation of the metabolic outcome measures, might account for small-scale variations in numeric results, thus requiring caution in rating device performance based only on findings on metabolic consumption. For example, in this study, the computation of gross rather than net metabolic power for the healthy subjects partly contributed to comparatively underestimate the gain in ExoAM. Furthermore, in our study, the choice of slower treadmill speeds than others exploiting similar orthoses and torque profiles might partly explain our relatively lower metabolic reductions: in fact, the higher range of motion and muscular activation observed during hip flexion/extension for increasing speed [36] might augment the beneficial effect of the assistance during fast walking. In line with this, other studies exploring treadmill speeds comparable to ours, also reported similar metabolic reductions [20], [37].

Overall, several open questions remain unexplored, such as the importance of providing assistive extensor torque on the prosthetic limb. In [34], [38], walking with a hip exoskeleton that provided assistance only during the extension phase was found to reduce the metabolic cost of walking in healthy subjects, but such an assistive strategy delivering purely extensor torques has never been tested in amputees. Regarding the assistive torque, it would be interesting to investigate whether a unilateral device extremely reduced in weight yet assisting only the prosthetic limb would be more effective than a bilateral one, which has a higher mass but can deliver up to double the power. Our results showing only a limited increase in metabolic consumption in ExoTM would support that a bilateral device could be worth to restore physiological patterns on the sound side as well as on the prosthetic side. For example, an assistive extension torque on the sound limb could mitigate amputees' increased hip work, probably a compensation for the decreased concurrent push-off on the prosthetic side [39], [40]. Moreover, a study comparing unilateral and bilateral assistance with an ankle exoskeleton, showed that metabolic consumption was maximally reduced with bilateral assistance, either splitting the total power provided unilaterally among the two limbs or doubling that amount replicating the unilateral assistance on both limbs [41]. These results would need to be verified with amputees. Nonetheless, the reduced weight of unilateral devices would be expected to elicit significant gains in gait energetics. Thus, reducing (and optimally distributing) the weight added by the exoskeleton might as well override the importance of assisting both limbs as a primary design specification for devices intended to reduce the energetic cost of walking.

\section{CONCLUSION}

In this study, a portable powered hip orthosis was tested with healthy subjects and two transfemoral amputees. Assisting hip flexion/extension with the exoskeleton was found effective to reduce the metabolic cost of treadmill walking for the amputees, whilst the overall beneficial effects for healthy subjects were observed to a lesser extent and did not overcome the burden of carrying the exoskeleton. Our results confirmed the potential effectiveness of exoskeletons to assist the gait of transfemoral amputees, but several open points need to be still investigated. Further assessments with amputees are needed to identify optimal assistive strategies and parameters as well as design guidelines for device optimization for these specific users.

\section{REFERENCES}

[1] L. van Schaik, J. H. B. H. Geertzen, P. U. Dijkstra, and R. Dekker, "Metabolic costs of activities of daily living in subjects with lower limb amputation: a systematic review and metaanalysis," PLoS One, vol. 14, no. 3, pp. 1-24, Mar. 2018.

[2] J. J. Genin, G. J. Bastien, B. Franck, C. Detrembleur, and P. A. Willems, "Effect of speed on the energy cost of walking in unilateral traumatic lower limb amputees," Eur. J. Appl. Physiol., vol. 103, no. 6, pp. 655-663, Aug. 2008.

[3] E. Russell Esposito, C. A. Rábago, and J. Wilken, "The influence of traumatic transfemoral amputation on metabolic cost across walking speeds," Prosthet. Orthot. Int., vol. 42, no. 2, pp. 214222, Apr. 2018.

[4] M. S. Pinzur, J. Gold, D. Schwartz, and N. Gross, "Energy demands for walking in dysvascular amputees as related to the level of amputation," Orthopedics, vol. 15, no. 9, pp. 1033-1037, 1992.

[5] R. L. Waters, J. Perry, D. Antonelli, and H. Hislop, "Energy cost of walking of amputees: the influence of level of amputation," $J$. Bone Jt. Surg. - Ser. A, vol. 58, no. 1, pp. 42-46, 1976.

[6] M. K. Ishmael, M. Tran, and T. Lenzi, "ExoProsthetics: Assisting Above-Knee Amputees with a Lightweight Powered Hip Exoskeleton," in 2019 IEEE 16th International Conference on Rehabilitation Robotics (ICORR), 2019, pp. 925-930.

[7] J. D. Smith and P. E. Martin, "Effects of prosthetic mass distribution on metabolic costs and walking symmetry," J. Appl. Biomech., vol. 29, no. 3, pp. 317-328, Jun. 2013.

[8] V. Monaco et al., "An ecologically-controlled exoskeleton can improve balance recovery after slippage," Sci. Rep., vol. 7, no. 1, pp. 1-10, May 2017.

[9] C. B. Sanz-Morère et al., "A Bioinspired Control Strategy for the CYBERLEGs Knee-Ankle-Foot Orthosis: Feasibility Study with Lower-Limb Amputees," in Proceedings of the IEEE RAS and EMBS International Conference on Biomedical Robotics and Biomechatronics, 2018.

[10] M. F. Eilenberg, K. Endo, and H. Herr, "Biomechanic and Energetic Effects of a Quasi-Passive Artificial Gastrocnemius on Transtibial Amputee Gait," J. Robot., vol. 2018, 2018.

[11] D. J. Farris and G. S. Sawicki, "The mechanics and energetics of human walking and running: A joint level perspective," J. R. Soc. Interface, vol. 9, no. 66, pp. 110-118, Jan. 2012.

[12] F. Giovacchini et al., "A light-weight active orthosis for hip movement assistance," Rob. Auton. Syst., vol. 73, pp. 123-134, Nov. 2015.

[13] J. H. Meuleman, E. H. F. Van Asseldonk, and H. Van Der Kooij, "The effect of directional inertias added to pelvis and ankle on gait," J. Neuroeng. Rehabil., vol. 10, no. 1, pp. 1-12, Apr. 2013. 
[14] T. Yan, A. Parri, V. Ruiz Garate, M. Cempini, R. Ronsse, and N. Vitiello, "An oscillator-based smooth real-time estimate of gait phase for wearable robotics," Auton. Robots, vol. 41, no. 3, pp. 759-774, Mar. 2017.

[15] R. Ronsse et al., "Oscillator-based assistance of cyclical movements: Model-based and model-free approaches," Med. Biol. Eng. Comput., vol. 49, no. 10, pp. 1173-1185, Oct. 2011.

[16] H. J. Lee et al., "A Wearable Hip Assist Robot Can Improve Gait Function and Cardiopulmonary Metabolic Efficiency in Elderly Adults," IEEE Trans. Neural Syst. Rehabil. Eng., vol. 25, no. 9, pp. 1549-1557, 2017.

[17] E. C. Martinez-Villalpando, L. Mooney, G. Elliott, and H. Herr, "Antagonistic active knee prosthesis. A metabolic cost of walking comparison with a variable-damping prosthetic knee," in Proceedings of the IEEE International Conference of Engineering in Medicine and Biology Society, EMBS, 2011, pp. 8519-8522.

[18] D. A. Winter, Biomechanics and Motor Control of Human Movement. 2009.

[19] G. J. Balady et al., "Clinician's guide to cardiopulmonary exercise testing in adults: A scientific statement from the American heart association," Circulation, vol. 122, no. 2. pp. 191-225, 13-Jul-2010.

[20] I. Kang, H. Hsu, and A. Young, "The Effect of Hip Assistance Levels on Human Energetic Cost Using Robotic Hip Exoskeletons," IEEE Robot. Autom. Lett., vol. 4, no. 2, pp. 430437, 2019.

[21] B. J. Whipp and K. Wasserman, "Oxygen uptake kinetics for various intensities of constant-load work.," J. Appl. Physiol., vol. 33, no. 3, pp. 351-356, 1972.

[22] J. M. Brockway, "Derivation of formulae used to calculate energy expenditure in man," Hum. Nutr. Clin. Nutr., vol. 41, no. 6, pp. 463-471, Nov. 1987.

[23] S. Kipp, W. C. Byrnes, and R. Kram, "Calculating metabolic energy expenditure across a wide range of exercise intensities: The equation matters," Appl. Physiol. Nutr. Metab., vol. 43, no. 6, pp. 639-642, 2018.

[24] B. M. Nes, I. Janszky, U. Wisløff, A. Støylen, and T. Karlsen, "Age-predicted maximal heart rate in healthy subjects: The HUNT Fitness Study," Scand. J. Med. Sci. Sports, vol. 23, no. 6, pp. 697-704, Dec. 2012.

[25] H. K. Hiilloskorpi, M. E. Pasanen, M. G. Fogelholm, R. M. Laukkanen, and A. T. Mänttäri, "Use of heart rate to predict energy expenditure from low to high activity levels," Int. J. Sports Med., vol. 24, no. 5, pp. 332-336, Jul. 2003.

[26] D. E. R. Warburton, C. Whitney Nicol, and S. S. D. Bredin, "Prescribing exercise as preventive therapy," Can. Med. Assoc. J., vol. 174, no. 7, pp. 961-974, 2006.

[27] E. Martini et al., "Gait training using a robotic hip exoskeleton improves metabolic gait efficiency in the elderly," Sci. Rep., vol. 9, no. 1, pp. 1-12, 2019.

[28] R. J. Nied and B. Franklin, "Promoting and prescribing exercise for the elderly," Am. Fam. Physician, vol. 65, no. 3, pp. 419-428, 2002.

[29] F. A. Panizzolo et al., "Metabolic cost adaptations during training with a soft exosuit assisting the hip joint," Sci. Rep., vol. 9, no. 1, pp. 1-10, Dec. 2019.

[30] S. F. Ray, S. R. Wurdeman, and K. Z. Takahashi, "Prosthetic energy return during walking increases after 3 weeks of adaptation to a new device," J. Neuroeng. Rehabil., vol. 15, no. 1, p. 6, Jan. 2018.

[31] A. T. Johnson, M. Benhur Benjamin, and N. Silverman, "Oxygen consumption, heat production, and muscular efficiency during uphill and downhill walking," Appl. Ergon., vol. 33, no. 5, pp. 485-491, 2002.

[32] G. S. Sawicki and D. P. Ferris, "Mechanics and energetics of incline walking with robotic ankle exoskeletons," J. Exp. Biol., vol. 212, no. 1, pp. 32-41, Jan. 2009.

[33] K. Seo, J. Lee, and Y. J. Park, "Autonomous hip exoskeleton saves metabolic cost of walking uphill," in IEEE International Conference on Rehabilitation Robotics, 2017, pp. 246-251.

[34] A. J. Young, J. Foss, H. Gannon, and D. P. Ferris, "Influence of Power Delivery Timing on the Energetics and Biomechanics of Humans Wearing a Hip Exoskeleton," Front. Bioeng. Biotechnol., vol. 5, no. March, pp. 1-11, Mar. 2017.
[35] K. Seo, J. Lee, Y. Lee, T. Ha, and Y. Shim, "Fully autonomous hip exoskeleton saves metabolic cost of walking," in Proceedings - IEEE International Conference on Robotics and Automation, 2016, vol. 2016-June, pp. 4628-4635.

[36] M. P. Murray, L. A. Mollinger, G. M. Gardner, and S. B. Sepic, "Kinematic and EMG patterns during slow, free, and fast walking," J. Orthop. Res., vol. 2, no. 3, pp. 272-280, 1984.

[37] R. Kitatani, K. Ohata, H. Takahashi, S. Shibuta, Y. Hashiguchi, and N. Yamakami, "Reduction in energy expenditure during walking using an automated stride assistance device in healthy young adults," Arch. Phys. Med. Rehabil., vol. 95, no. 11, pp. 2128-2133, Nov. 2014.

[38] Y. Ding et al., "Effect of timing of hip extension assistance during loaded walking with a soft exosuit," J. Neuroeng. Rehabil., vol. 13, no. 1, pp. 1-10, 2016.

[39] R. E. Seroussi, A. Gitter, J. M. Czerniecki, and K. Weaver, "Mechanical work adaptations of above-knee amputee ambulation," Arch. Phys. Med. Rehabil., vol. 77, no. 11, pp. 1209-1214, 1996.

[40] T. S. Bae, K. Choi, D. Hong, and M. Mun, "Dynamic analysis of above-knee amputee gait," Clin. Biomech., vol. 22, no. 5, pp. 557-566, Jun. 2007.

[41] P. Malcolm, S. Galle, P. Van Den Berghe, and D. De Clercq, "Exoskeleton assistance symmetry matters: Unilateral assistance reduces metabolic cost, but relatively less than bilateral assistance," J. Neuroeng. Rehabil., vol. 15, no. 1, pp. 1-11, 2018. 